\title{
Practitioner Perceptions of the Health of Australian First Nations'
}

\section{Peoples: Preliminary Findings}

Alan Crouch (The University of Melbourne)

$60 \%$ (contributions to conception and design of the work; acquisition, analysis and interpretation of data; drafting author; final approval for submission for publication; agreement to be accountable for all aspects of the work in ensuring that questions related to the accuracy or integrity of any part of the work are appropriately investigated and resolved.)

Bonnie Chew (Mirriyu Cultural Consulting)

20\% (final approval for submission for publication; critical revision for content and cultural safety; final approval for submission for publication agreement to be accountable for all aspects of the work in ensuring that questions related to the accuracy or integrity of any part of the work are appropriately investigated and resolved.)

Gwenda Freeman (The University of Melbourne)

$20 \%$ (analysis and interpretation of data; final approval for submission for publication; agreement to be accountable for all aspects of the work in ensuring that questions related to the accuracy or integrity of any part of the work are appropriately investigated and resolved.)

Corresponding Author:

Name: Alan Crouch

Address: The University of Melbourne Department of Rural Health, 806 Mair Street Ballarat VIC 3350

Email: Alan.Crouch@unimelb.edu.au

Phone: 0419573728

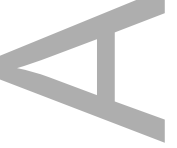

This is the author manuscript accepted for publication and has undergone full peer review but has not been through the copyediting, typesetting, pagination and proofreading process, which may lead to differences between this version and the Version of Record. Please cite this article as doi: $10.1111 /$ AJR.12645

This article is protected by copyright. All rights reserved 


\section{Disclosure Statement:}

This research was funded by the University of Melbourne Department of Rural Health. The paper was presented at the $15^{\text {th }}$ National Rural Health Conference in Hobart, Tasmania in March 2019.

\section{Acknowledgements}

The authors wish to acknowledge the support of the numerous health discipline professional organisations and associations through which access to the survey underpinning this paper was circulated to respective practitioner and student members.

\section{Conflict of Interest}

Alan Crouch and Gwenda Freeman declare no conflict of interest in this work. Bonnie Chew declares that she was the developer and facilitator of an Aboriginal cultural awareness training program and that a small proportion of survey respondents $(<10 \%)$ completed this training program. Ms Chew, who has not had access to any unprocessed survey data, wishes to use the findings of the study to further develop and refine her approach to cultural awareness training.

\section{Ethic Approval}

Ethical approval for this study, with an Ethics ID 1647148.2, was endorsed by the Melbourne Medical School Human Ethics Sub-Committee.

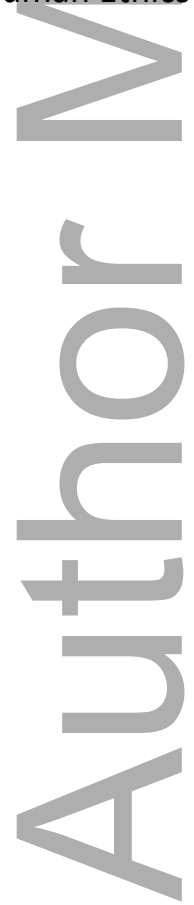

This article is protected by copyright. All rights reserved 


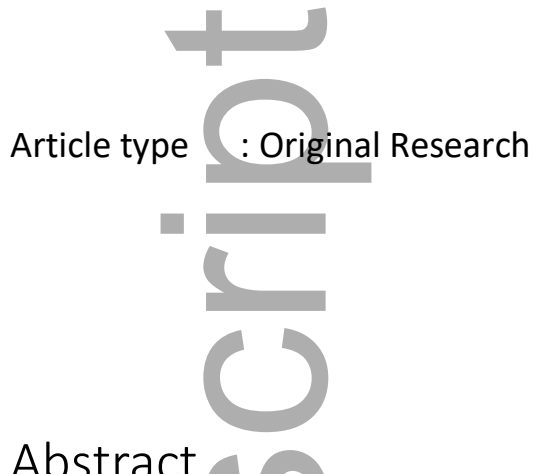

Abstract

Objective: To probe health practitioner perceptions of Australian First Nations' Peoples' health and to compare these with reported burden of disease, social determinants and culturally safe health systems data.

Design: An on-line survey tool was used to collect self-reported responses from health practitioners to answer literature-derived questions regarding underlying issues in Australian First Nations' Peoples' health.

Setting: Responses to the survey were sought from rural and urban health-service locations across all Australian States and Territories

Participants: Allied health, nursing, pharmacy, psychology, social work and related discipline students and practitioners currently working or studying in Australia

Main outcome measure(s): Degrees of correspondence between health discipline student and practitioner perceptions on 'major health issues' and 'health systems issues' and published population health and health systems data. Metrics for: ' connectedness to' and 'preparedness to engage with' Australian First Nations' Peoples were also reported.

Results: Significant differences between practitioner perceptions of 'major health issues' and the disease burden / social determinants published evidence, and with the 'culturally safe health systems' published evidence, were noted. Positive impacts of social and professional relationships (connectedness) between practitioners and First Nations' Peoples were demonstrated.

Conclusions: The inclusion of basic population health and culturally safe health systems training in curricula for all genres of health practitioners appears to be indicated by these findings. Further, a meaningful role for the intentional nurture of social and professional relationships with Australian 
First Nations' Peoples across all health disciplines is suggested as part of efforts to address health systems and equity issues.

\section{Keywords}

Australian First Nations' Peoples'cultural awareness; cultural safety; burden of disease; social determinants; culturally safe health systems;

\section{Boxed Lists}

What is already known on this subject

Cultural awareness training alone is not an effective strategy in producing change or achieving better healthcare outcomes for Australian First Nations' Peoples and is ineffective in changing attitudes and beliefs when delivered in isolation or rapidly delivered over short timeframes.

Cultural awareness training is more effective for health practitioners having familiarity or friendships with Australian First Nations' Peoples, in terms of understanding the complexity of health issues

In Australia, there is a lack of evidence about the degree to which health practitioner perceptions of Australian First Nations' Peoples' health correspond with the data on disease burden, social determinants and cultural safety of health systems

\section{What this paperadds}

There appears to be a significant difference between practitioner perceptions of major issues in First Nations' Peoples' health and the social determinants and epidemiological data

There appears to be a significant difference between practitioner perceptions of health systems issues impacting Australian First Nations' Peoples health and health systems parameters supporting wellbeing identified by Australian First Nations' Peoples

Social and professional interaction between individual health practitioners and Australian First Nation's Peoples appears to strengthen practitioner preparedness to engage with, advocate for and promote equity in health for Australian First Nations' Peoples. 
The findings have implications for approaches to pre-service and in-service education and training, to include basic units in population health and health system cultural safety across the spectrum of health disciplines and professions

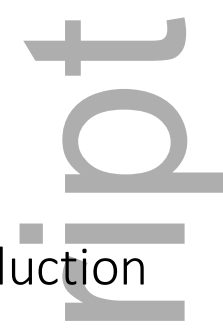

Cultural awareness trainingof health practitioners is one of manystrategies appliedto address health inequities across multicultural populations. However, the literature on the effectiveness of this training in influencing professional practice and health outcomesis inconclusive, in part related to the diversity of benchmarks against which 'effectiveness' has been assessed(1). Some approaches have sought to use clinical outcomes as a direct measure of training effectiveness [2-3]. Others have sought to distinguish between perceptions, attitudes and practices among health service staffas indirect measures of training effectiveness (2-4). Yet others have explored 'hospital culture', using a continuous quality improvement framework to identify structural factors influencing training efficacy and patient outcomes (5).

In general, alack of clarity in the literature on the relationship between culture, cultural awareness training of health practitioners and clinical and population health outcomes has been noted(6).A systematic review, focusing on clinical outcomes as the benchmark of training effectiveness,sought unsuccessfully to identify 'dose-response' relationships between cultural training and patient outcomes (7). A studyfocusing on practitioner attributes as proxies for practice intentionsdescribed a relationship between training duration and effectiveness. This study reported that less than a halfday exposure to cultural trainingwas ineffective in changing practitioner beliefs and attitudes(4).

A study on undergraduate medical students reported that the integration of cultural awareness training into pre-service education improved student knowledge and skills, and in this context, increased both student preparedness to work with and care for Australian First Nations' Peoples and student willingness to advocate for and improve First Nations' Peoples' health (8). The study also found that the presence of existing relationships between health practitioners and persons identifying as of First Nations' heritage increased the likelihood that training participants would experience empathetic belief and attitudinal changes, as well as increasing understanding of the complexity of First Nations' Peoples health issues(8). These findings were also reflected in a study of undergraduate midwifery students, noting that the inclusion of an undergraduate unit on Aboriginal 
health enhanced student knowledge and shifted attitudes toward Aboriginal Peoples in a positive direction(9)

Evidence from health service planning strongly suggests that the concurrent implementation of a range of strategic approaches is required to achieve equitable health outcomes across different population groups(10). However, the practice of and place for cultural awareness training and related training, as well as any implications forcultural safety(11),generally remains inadequately evinced despite widespread implementation. This is specifically soin the context of currentholistic, multi-strategic approaches to address historical health inequities among Australian First Nations'Peoples(12).

Given the national priority for First Nations' Peoples' health equity across urban, rural and remote settings and the importance of a culturally aware and safe workforce(13), it is the aim of this study to explore more deeply the knowledge, understandings, attitudes and beliefs of health practitioners and health-discipline students regarding Australian First Nations' Peoples wellbeing. This, it is posited, is a critical step towards improving the cultural appropriateness of health service planning and provision. Ethical approval for this study, with an Ethics ID 1647148.2, was endorsed by the Melbourne Medical School Human Ethics Sub-Committee.

\section{Methodology}

A mixed methods approach was taken to study data collected throughan on-line survey of healthdiscipline practitioners and students who had ever in their careers or studies undertaken Australian First Nations' Peoples cultural awareness training of any duration. The survey contained twenty-one questions, fourteen of which were open-ended, requiring free text responses. Survey questions, framed in the context offindings documented in peer-reviewed studiesof training effectiveness, elicited demographic data, free text data on health and health systems Issues, as well as selfreported degrees, respectively, of preparedness to engage with First Nations'Peoples, willingness to advocate for health equity and readiness to actively promote health system change. Survey questions also sought responses on the duration of training and the degree of respondent connectedness at professional and social levels, with Australian First Nations' Peoples.

Study data were collected and managed using REDCap electronic data capture tools hosted the University of Melbourne. The survey was administered over an eighteen-month period in 2017 and 2018. There were two related but separate pathways to survey participation.Links to the survey were circulated to student and graduate members of professional associations across the spectrum of nursing, allied health, pharmacy, psychology, social work and related disciplines through electronic newsletters, social media platforms and email systems. A total of eight allied health and 
nursing professional associations agreed to distribute the survey link to practitioner and student members. It was not possible to estimate the potential numbers of participants, due to the indeterminate numbers from each participating professional organisation who actually read the electronic newsletters or other digital media and made a decision on whether or not to access the survey link. The link was also sent directly to approximately 120 health practitioner staff and student participants in a University of Melbourne cultural awareness training program, who had provided email addresses for evaluation purposes. Descriptive data were analysed for content, using both inductive and deductive methods(14). Inductive content analysis was undertaken by exporting all free text data fields containing responses (including null responses) to each question from the REDCap survey database into question-specific Microsoft Word ${ }^{\mathrm{TM}}$ documents. Initial coding, based on text intrinsic to the responses for each question, was conducted by one author. All authors then reviewed and revised where indicated the initial coding for responses to each free text question and agreed on the major themes and subthemes described and on their occurrence in the data $(15,16)$.

Deductive content analysis was undertaken similarly, using terms (and their synonyms) extrinsic to the data, identified in an environmental scan of published disease burden, social determinants and health systems literature. The question-specific Microsoft Word ${ }^{\mathrm{TM}}$ documents were searched for occurrences of the identified terms and their synonyms (as text strings), with each occurrence of the string contextually validated for relevance and logical consistency.

Numeric data were analysed using fit-for-purpose statistical tests within the IBM SPSS Statistics for Windows, version 24.0 software package, including the Paired-Sample T test for difference of means and the Mann-Whitney U Test.

Results

The preliminary findings from the analyses of survey data (see below for detail) include geographic, age and duration of cultural awareness training distributions of respondents, together with the outcomes of content analysis on the free text responses to questions regarding major health and health systems issues impacting the health and wellbeing of First Nations' Peoples. Preliminary findings on the degree of 'preparedness' of health practitioners to engage with First Nations' Peoples and the degree to which health practitioners had social or professional relationships with First Nations' Peoples are also reported.

\section{Demographic Characteristics of Respondents}

Respondents to the survey ( $\mathrm{N}=131$ at the time of the preliminary analysis) were drawn from all Australian States and mainland Territories, with the majority residing in Western Australia (25\%), 
New South Wales (24\%), Victoria (22\%) and Queensland (16\%).Other states and territories accounted for $13 \%$ of respondents.

In terms of age range proportions, respondents included: $18-24$ years (5\%); $25-34$ years (32\%); 35-44 years (18\%); $45-54$ years (31\%); $55-64$ years (17\%); $65-74$ years $(4 \%)$, and;>75 years( $2 \%)$.

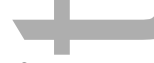

In terms of cultural awareness training duration, the proportions were: 1-2 hours (14\%); 2-4 hours (21\%); 4-6 hour (10\%); one working day (30\%), and;>1 working day (27\%).

In terms of health practitioner connectedness (professional or social) to Australian First Nations' Peoples, approximately one half of respondents, in answering a multiple-choice question on numbers of contacts, reported 'No-one' and approximately one half selected options indicating one or more $\operatorname{contacts}(n=80)$.

\section{Content Analysis}

Inductive content analysisof free text responses to the question: "What, in your understanding, are the major health issues facing people in Australia who identify as Aboriginal or Torres Strait Islander?", in which themes were suggested by the text itself, identifiedterms signifying: mental illness; cardiovascular disease; respiratory disease; skin disease; renal disease; endocrine disease; cancer; alcohol related disease; infections; obesity; malnutrition; diseases of the eye, ear, nose and throat, and; injuries. These free-text terms were codified accordingly as themes, and thesethemes were aggregated into the category 'Diseases' $(n=76)$. The analysis of responses to this question also identified a range of terms signifying structural, cultural, social and systemic factors influencing First Nations' Peoples' health, including: access; racism; socio-economic status; health literacy; culture, and; a range of other factors. These terms were codified accordingly as themes and aggregated into the category 'Social Determinants' $(n=29)$.

Inductive content analysisof free text responses to the question: "What do you imagine are the key health systems issues impacting on Aboriginal and Torres Strait Islander health?" identified terms signifying: mistrust and unwelcoming spaces; stigma, discrimination and racism; culturally insensitive practice; lack of Indigenous staff, and service delivery and barriers to care. These free text terms were codified accordingly as themes and aggregated into the category 'Culturally Safe Health Care'.

The occurrence of themes within each category was determined by word count of free text terms associated with the respective theme (Table 1). 
Interpretive Frameworks for Deductive Content Analysis

An environmental scan of published literature related to each of the broad categories described through inductive contentanalysis (Disease, Determinants and Culturally Safe Health Care) was undertaken to identify benchmark frameworks for each category, with a focus on works produced by scholars, practitioners and agencies identifying as Australian First Nations, as well as research consortia led or guided by Australian First Nations'Peoples. For Diseases, the Australian Burden ofDisease Study: impact and causes of illness and death in Aboriginal and Torres Strait Islander people 2011(17) was identified and required no further development to be fit-for-purpose for this study. Similarly, the Kanyini Vascular Collaboration Wellbeing Framework(18) required only minor adaptation for use as a benchmark for Culturally Safe Health Care. The Social Determinants framework, however was synthesised from the key factors identified in each of the three comprehensive studies on the determinants of Australian First Nations'Peoples'health identified through the environmental scan (19-21).

Deductive content analysis, in which the themes were suggested by the respective reference framework categories, was then undertaken on free text survey data for each of the Major Health Issues (see Figure1 and Table 2) and Health Systems Issues fields (see Table 3). The findings were then compared with respective reference framework evidence to gauge the degree of correspondence with respondents' reported perceptions.

\section{Discussion}

\section{Demographics}

Respondents to the survey included people across the age spectrum who were drawn from all Australian states and mainland territories, and who were student and graduate members of professional associations across the spectrum of nursing, allied health, pharmacy, psychology, social work and related disciplines, indicating the broad reach and professional diversity among survey respondents.

\section{Major Health Issues - Diseases}

Survey respondent perceptions of major health issues were mapped against the eight highest causes of fatal disease burden in Australian First Nations' Peoples(Figure 1). For the purpose of determining proportions in this exercise, the denominator selected was all survey respondents $(N=131)$, whether or not text was added to the particular survey field, on the basis that a null result was not an insignificant finding. Significant gaps in the knowledge of health practitioners were identified. Less than one third of respondents identified the disease group causing the highest fatal burden (mental health / substance use). Around one quarter of respondents identified cardiovascular disease and 
stroke, causing the third highest disease burden. A similar number identified endocrine disease (mainly diabetes), causing the eighth-highest disease burden. However, less than $5 \%$ of respondents identified injuries (including suicide), cancer (which is twice the disease burden of diabetes), respiratory conditions, musculoskeletal diseases and infant and congenital conditions. It should be noted that each one of these disease groups causes a higher fatal burden than endocrine diseases (mainly diabetes), yet practitioner perceptions of diabetes importance ranked this disease as second most important.

Major Health Issues -Social Determinants

A similar picture emerged when survey respondent perceptions of the determinants of health $(n=131)$ were mapped against the benchmark framework of nine key determinants synthesised from the three comprehensive studies identified (Table 2). Only one determinant of health defined by First Nations' Peoples was identified by $>10 \%$ of survey respondents - interaction with government systems. Typically, this was associated with health service access issues in responses. Importantly, only two respondents in this sample identified community control of health services as a key determinant in response to the Major Health Issues question. This may signifya need for more exposure in pre-and in-service trainingof health practitioners to the Australian First Nations' Peoplessocial determinants discourse.

Key Health Systems Issues

The Wellbeing Framework developed by the Kanyini Vascular Collaboration(18), based on core values for 'wellbeing' including '...upholding peoples' identities in connection to culture, spirituality, families, communities and country... supported by culturally safe primary healthcare services' defined four principal domains in describing the attributes of those healthcare services: 1 ) locally defined, culturally safe healthcare services; 2 ) appropriately skilled culturally competent healthcare team; 3) holistic care throughout the life span, and; 4) best practice care that addresses the particular needs of a community. Under each of these domains were defined four elements detailing key concepts in the respective domain, resulting in a sixteen-element matrix describing culturally safe primary healthcare services. Survey respondent perceptions of key health systems issues were mapped against the elements of the Wellbeing Framework (Table 3). This analysis again identified the low levels of correspondencebetween survey respondent health practitioner perceptions with the key health systems parameters described by researchers who identified as Australian First Nation Peoples. The only element identified by more than $10 \%$ of the respondents was service availability, accessibility and acceptability, consistent with findings from the analysis of social determinants data. The next most frequently identifieddomains include 'Culturally welcoming places'for service delivery (9\%)and 'valuing and supporting Australian First Nations'staff members' in 
the health service workplace (8\%), againconsistent with findings from the analysis of the social determinants data (Table 2). Notably, cultural diversity within communities and cultural and scientific best practice primary healthcare were not identified by any of the respondents in the sample.

These findings around practitioner perceptions of major health issues impacting on Australian First Nations' Peopleshighlight the need for greater population health knowledge in the health practitioner workforce. Similarly, the findings around key health systems issues speak to a need for a better process of practitioner orientation to well-functioning health systems in general and to those system factors in particular limiting Australian First Nations' Peoples'engagement with government andprivate health services.

Health Practitioner Preparedness to Engage

Survey respondents were asked to report their perceptions of 'preparedness to engage' with Australian First Nations' People as patients / clients, both before and following any cultural awareness training of any duration. The degree of preparedness was expressed using a sliding scale, with a value assigned by the REdCap software based on the movement of the scale pointer from the resting position (Figure 2).

The findings indicate a statistically significant $(P<.001)$ difference between respondent pre- and postrecollected perceptions of 'Preparedness to engage' (using the Paired-Sample T test for difference of means), suggestive of positive attitudinal change following cultural awareness training. This is an important observation supporting the continuation of investment in cultural awareness training, given the current lack of evidence for effectiveness.

A statistically significant relationship between 'Preparedness to Engage' and 'Duration of Training' data was also found in this preliminary analysis (Mann-Whitney U Test; significance level=.037). These data appear to supportthe 2006 findings of an earlier study(4)that cultural awareness training is less effective in changing attitudes and beliefs when delivered rapidly over short timeframes.

When 'Connectedness to Australian First Nations' Peoples values for respondents reporting $75 \%$ or greater 'Preparedness to engage' were determined $(n=31), 27$ respondents selected options in the multi-choice question indicating one or more contacts, and only 4 selected 'No-one'. Thispreliminary evidence of the positive effect of connectedness is suggestive of the critical importance of relationships between health practitioners and Australian First Nations' People in both improving the effectiveness of cultural awareness training and in actually addressing health inequity. This 
reality has long been appreciated and promoted by First Nations People-advocates, as is evinced in the Wellbeing Framework developed by the Kanyini Vascular Collaboration Wellbeing Team.

\section{Conclusions}

The most important national-level preliminary finding of this study is the disconnect between health practitioner knowledgeof major health issues and key health systems issues, across the spectrum of allied health, nursing, pharmacy, psychology, social work and related disciplines, and the published evidence of disease burden, determinants of health and well-functioning health systems. This further suggests that pre-service exposure to basic population health science and to well-functioning health systems theory should be embedded into the curricula of all genres of health practitioner undergraduate training in Australia.

A second preliminary observation supportsearlier findings that the duration of cultural awareness training impacts on its effectiveness, with less than one full day ineffective in changing attitudes and beliefs.

The final important preliminary observation made from the survey data is the impact of social and professional relationships between health practitioners and Australian First Nations' Peoples. This insightsuggests a meaningful role for intentional nurture of social and professional relationships in addressing the long-standing health inequity experienced by Australian First Nations' Peoples.

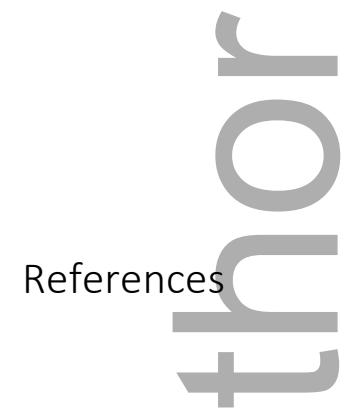

1. Bainbridge R, McCalman J, Clifford A, Tsey K. Cultural competency in the delivery of health services for Indigenous people. Canberra: Australian Institute of Health and Welfare; 2015. p. 1-44. 2. Chapman R, Martin C, Smith T. Evaluation of staff cultural awareness before and after attending cultural awareness training in an Australian emergency department. International Emergency Nursing. 2013;22 (4):179-84.

3. Flood JL, Commendador KA. Undergraduate nursing students and cross-cultural care: A program evaluation. Nurse Education Today. 2016;36:190-4. 
4. Mooney N, Bauman A, Westwood B, Kelaher B, Tibben B, Jalaludin B. A Quantitative Evaluation of Aboriginal Cultural Awareness Training in an Urban Health Service. Aboriginal and Islander Health Worker Journal. 2005(4):23.

5. Chong A, Renhard R, Wilson G, Willis J, Clarke A. Improving Cultural Sensitivity to Indigenous People in Australian Hospitals a Continuous Quality Improvement Approach. Focus on Health Professional Education: A Multi-disciplinary Journal. 2011(1):84.

6. Thackrah RD, Thompson SC. Refining the concept of cultural competence: building on decades of progress. Medical Journal of Australia 2013;199(1):35-8.

7. Lie DA, Lee-Rey E, Gomez A, Bereknyei S, Braddock CH. Does Cultural Competency Training of Health Professionals Improve Patient Outcomes? A Systematic Review and Proposed Algorithm for Future Research. Journal of General Internal Medicine. 2010(3):317-25.

8. Paul D, Carr S, Milroy H. Making a difference: the early impact of an Aboriginal health undergraduate medical curriculum. Medical Journal of Australia. 2006;184 (10):522-5.

9. Thackrah RD, Thompson SC, Durey A. Exploring undergraduate midwifery students' readiness to deliver culturally secure care for pregnant and birthing Aboriginal women. BMC Medical Education. 2015;15:77-86.

10. Hunter New England Health Aboriginal and Torres Strait Islander Strategic Leadership Committee. Closing the gap in a regional health service in NSW: a multi-strategic approach to addressing individual and institutional racism. New South Wales Public Health Bulletin. 2012;23 (34):63-7.

11. Bin-Sallik M. Cultural Safety: Let's Name It! The Australian Journal of Indigenous Education. 2003:21.

12. Department of Prime Minister and Cabinet. Closing the Gap: Prime Minister's Report. Canberra: Government of Australia; 2018.

13. Rural Health Standing Committee. National Strategic Framework for Rural and Remote Health. Standing Council on Health; 2011.

14. Graneheim UH, Lindgren B-M, Lundman B. Methodological challenges in qualitative content analysis: A discussion paper. Nurse Education Today. 2017;56:29-34.

15. Charmaz K. Constructing grounded theory : a practical guide through qualitative analysis: SAGE; 2006.

16. Whiteside M, Bould E, Tsey K, Venville A, Cadet-James Y, Morris ME. Promoting Twenty-firstcentury Student Competencies: A Wellbeing Approach. Australian Social Work. 2017;70(3):324-36. 17. Fadwa A-Y. The Australian Burden of Disease Study: impact and causes of illness and death in Aboriginal and Torres Strait Islander people, 2011. Public Health Research \& Practice. 2017(4). 
18. Davy C, Kite E, Sivak L, Brown A, Ahmat T, Brahim G, et al. Towards the development of a Wellbeing Model for Aboriginal and Torres Strait Islander peoples living with chronic disease BMC Health Services Research. 2017(17:659).

19. Australian Human Rights Commission. Information Sheet - Social justice and human rights for Aboriginal and Torres Strait Islander peoples. Canberra: Government of Australia; 2003.

20. Carson B, Dunbar T, Chenhall R, Bailie R, editors. Social Determinants of Indigenous Health. Sydney: Allen and Unwin; 2007.

21. Osborne K, Baum F, Brown L. What works? A review of actions addressing the social and economic determinants of Indigenous health. Canberra: Australian Institute of Health and Welfare; 2013.

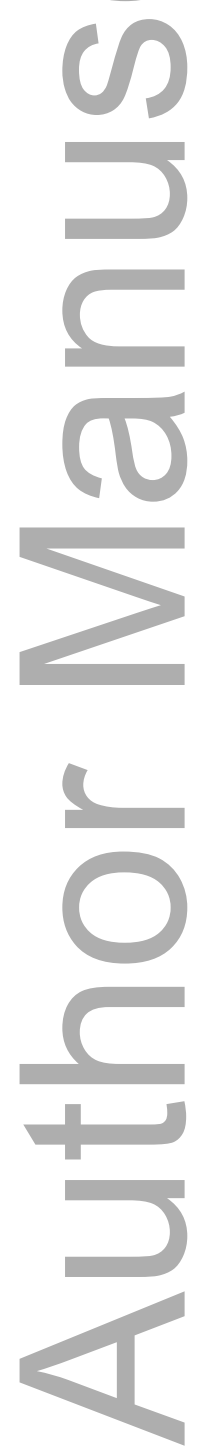


Tables

Table 1 Inductive Analysis Outcomes: Disease, Determinants and Health Systems Themes

\begin{tabular}{lc}
\hline DISEASE THEME & \multicolumn{2}{l}{ OCCURRENCE IN RESPONSES (\%) } \\
Disease & $55 \%$ \\
Mental health / substance use & $45 \%$ \\
Diabetes & $39 \%$ \\
Cardiovascular & $39 \%$ \\
Other chronic diseases & $17 \%$ \\
Infections & OCCURRENCE IN RESPONSES (\%) \\
DETERMINANTS THEME & \\
Determinant & $32 \%$ \\
Access & $18 \%$ \\
Racism & $10 \%$ \\
Socio-economic status & $14 \%$ \\
Health Literacy & $20 \%$ \\
Culture & $6 \%$ \\
Other & \\
HEALTH SYSTEM THEME & \\
Culturally safe health care & $31 \%$ \\
Mistrust and unwelcoming spaces & $29 \%$ \\
Stigma, discrimination and racism & $40 \%$ \\
Culturally insensitive practice & $19 \%$ \\
Lack of Indigenous staff & $44 \%$ \\
Service delivery and barriers to care & \\
&
\end{tabular}

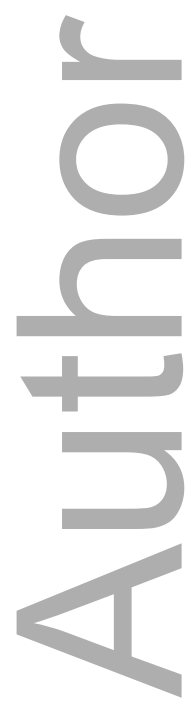

This article is protected by copyright. All rights reserved 


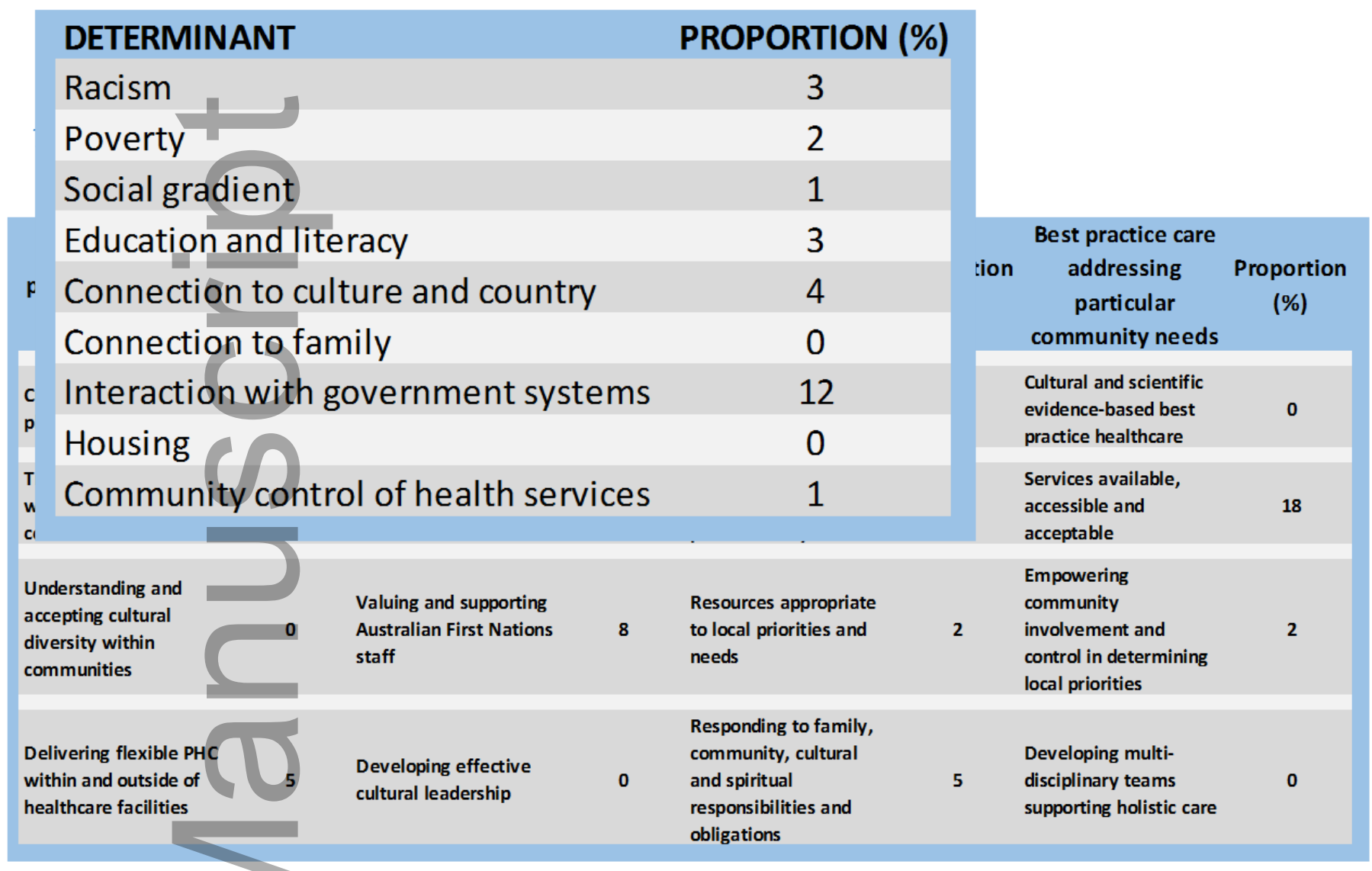

Adapted from the Kanyini Vascular Collaboration Wellbeing Framework (17)

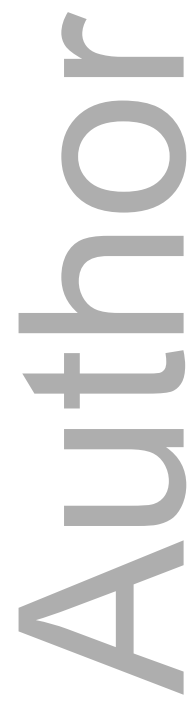

This article is protected by copyright. All rights reserved 
Figures

Figure 1 - Respondent Perceptions vs Disease Burden
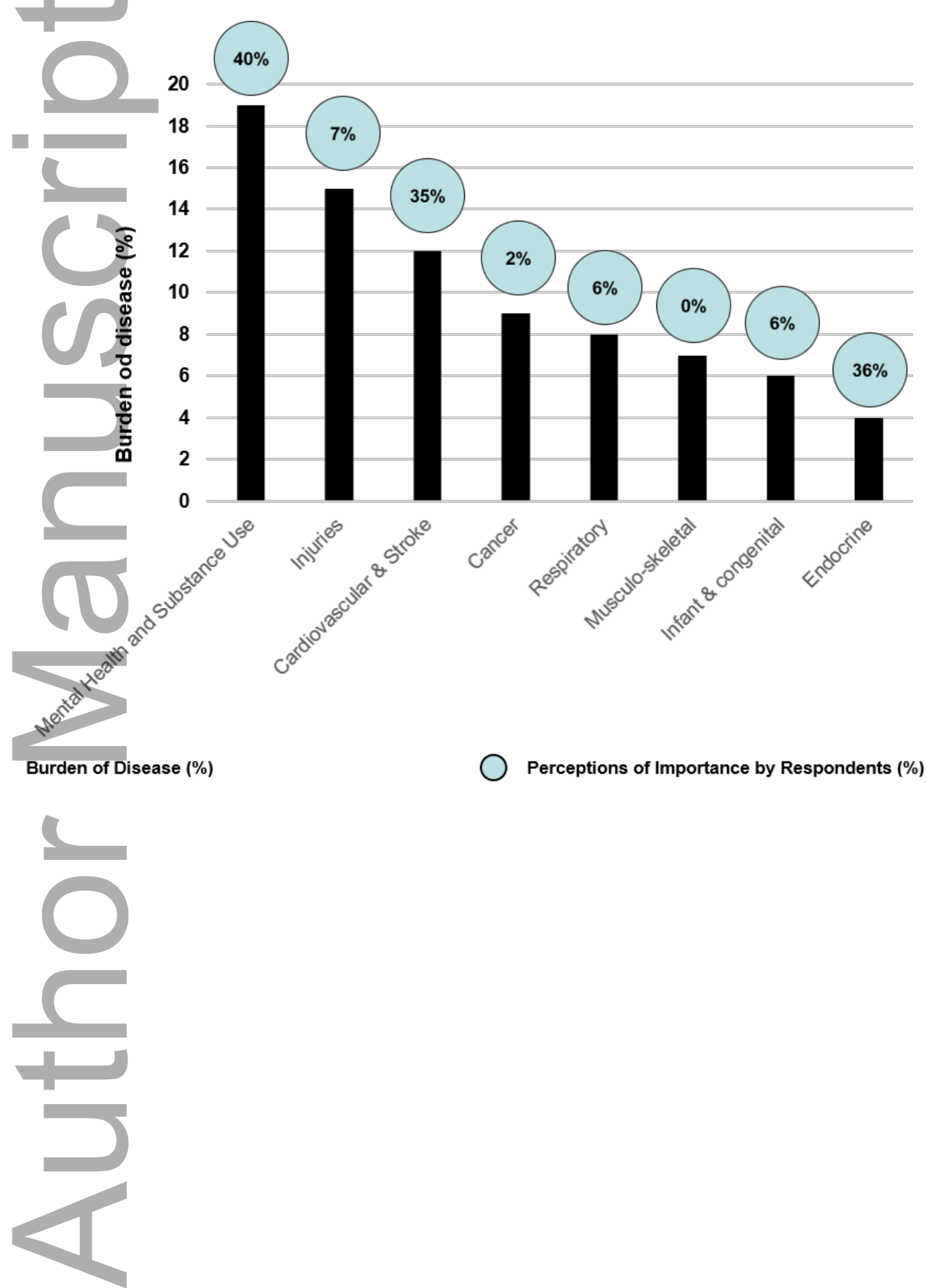

This article is protected by copyright. All rights reserved 
Figure 2-Respondent Recollected Perceptions of Preparedness: Pre and Post Training

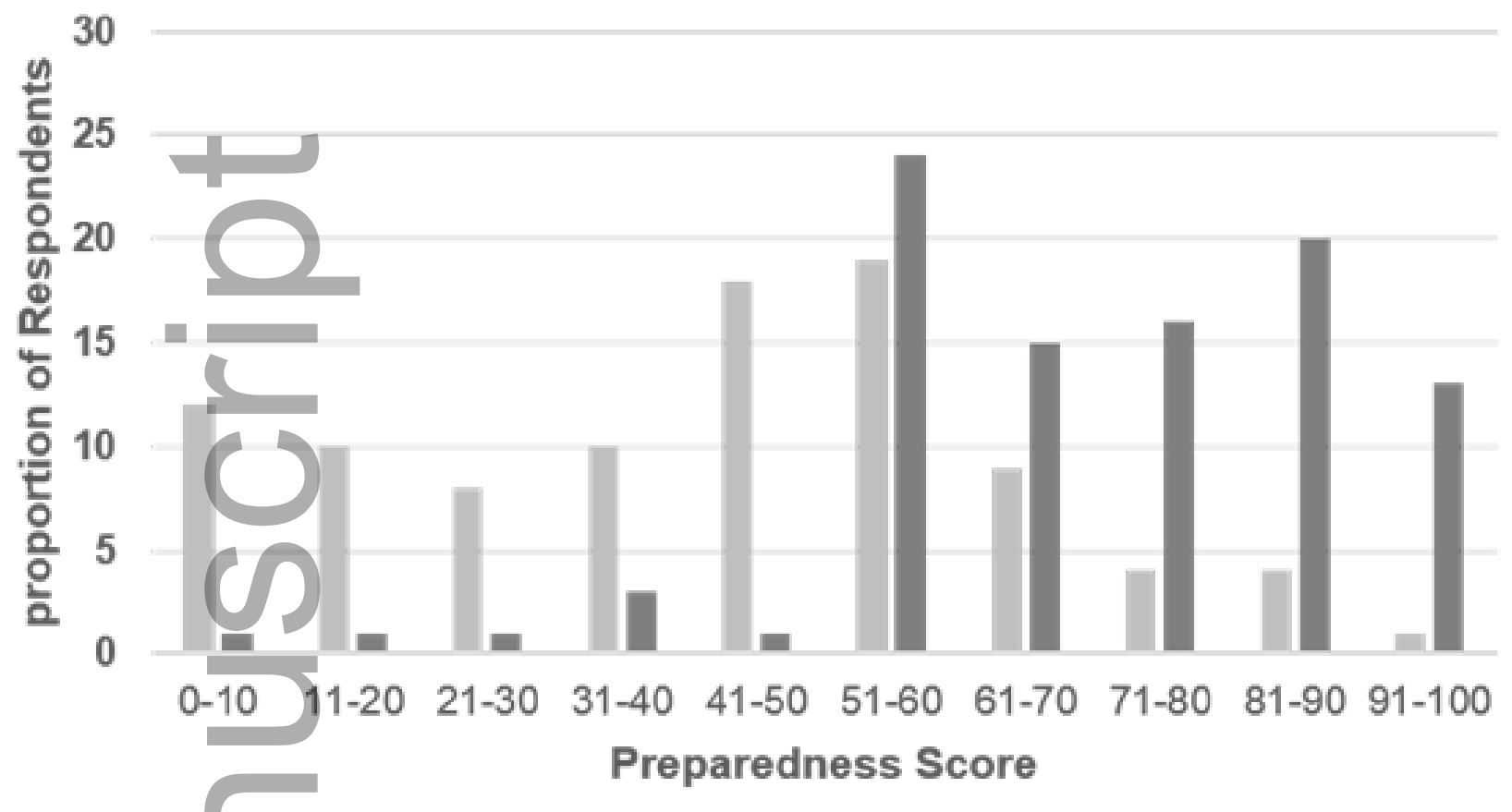

Pre-Training Preparedness Score $=$ Post-Training Preparedness Score (1)
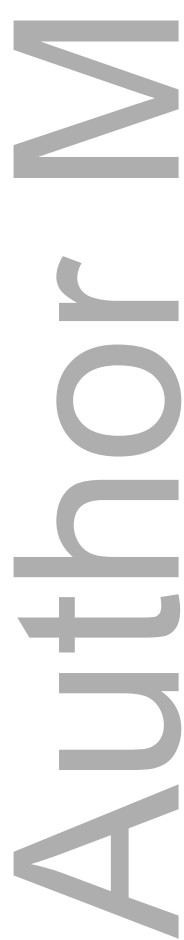

This article is protected by copyright. All rights reserved 


\section{University Library}

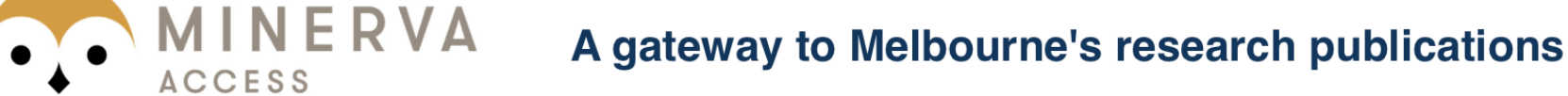

Minerva Access is the Institutional Repository of The University of Melbourne

Author/s:

Crouch, A;Cert, BC;Freeman, G

Title:

Practitioner perceptions of the health of Australian First Nations' Peoples: Preliminary findings

Date:

2020-07-30

\section{Citation:}

Crouch, A., Cert, B. C. \& Freeman, G. (2020). Practitioner perceptions of the health of Australian First Nations' Peoples: Preliminary findings. AUSTRALIAN JOURNAL OF RURAL HEALTH, 28 (4), pp.351-359. https://doi.org/10.1111/ajr.12645.

Persistent Link:

http://hdl.handle.net/11343/276077 